\title{
POWER LINE COMMUNICATION AN ESSENTIAL TOOL FOR DIGITAL BANGLADESH
}

\author{
Cdr M Mahbubur Rahman, BN ${ }^{(1)}$ and Md. Eliasinul Islam ${ }^{(2)}$
}

3. Department of Electrical Electronic and Communication Engineering, Military Institute of Science and Technology, E-mail: mr.eece@mist.edu.bd, mahbub549@yahoo.com

4. Department of Electrical Electronic and Communication Engineering, Military Institute of Science and Technology, E-mail: faded_elias@yahoo.com

\begin{abstract}
An estimation for the power line communications (PLC) for Bangladesh is made referring various technical research papers and current Journals. The aim of this study is to identify essential aspect of the power line communications for both domestic and outdoor application and the use of consumer communication when various possible services are developed worldwide commercially [1,9]. Possible services, bandwidth, service area, quality, reliability, and the cost are also taken into considerations. The signal in a transmission line is impaired by the presence of unwanted extraneous signals (noise and interference) on the line. The frequency dependent characteristics of the transmission line are also responsible to impair the transmission of signals along the lines. Noise and interference, with its presence tends to impede the reception of the desired signal and is usually the limiting factor in its detection. Noise is usually composed of randomly occurring voltages, which are unrelated in phase or frequency and may sometimes be of a very peak in nature. Interference on the other hand is usually more structured than noise since it arises as unwanted coupling from just a few signals in the network. In this paper a brief discussion of noise in a power line environment and need for power line communication in Bangladesh is presented as an essential tool for digital Bangladesh.
\end{abstract}

KEY WORDS - Cyclostationary noise; Attenuation; PDF(probability density function); White noise; Interference; Variance;etc.

\subsection{INTRODUCTION}

In the 1990s, companies such as Nortel Networks and Siemens recognized the potential of carrying Broadband communications over power-lines. They launched R\&D initiatives to send IP packets over power grids. But technology at the time faced hurdles. For example, to transmit data along noisy electric lines, the signals had to be turned up so high that they interfered with transmissions from other devices such as radios and military equipment. That is why, means to pass the broadband signal through the power transformer between the medium voltage (2.4-35 KV) and low voltage $(100-600 \mathrm{~V})$ could not be found ${ }^{[8]}$.

In the past, power line communication techniques were used for voice communication over long distance power transmission lines. However, as power transmission networks grew larger and became more complex and it became necessary to send and receive various information in addition to voice, such as supervisory data and commands for remote control between power plant and control center, separate communication networks were constructed using micro-wave and optical fiber technology. Nowadays, electric utilities do not use PLC over transmission lines; but some do use it over power distribution networks to reconfigure the networks and to measure power consumption in each home is usually confined to lower frequencies. This noise does not propagate very far from the source because it is a lowcurrent phenomenon that does not couple into the adjacent wires.

Based on this view point we consider it important to have an overall view for the application fields of the power-line communications at this stage.

\subsection{THE TECHNOLOGY ENVIRONMENT FOR PLC}

The power grid is a very hostile environment for higher frequency transmission. The overhead power lines are not insulated and no impedance matching is possible over the broad bandwidth (2$80 \mathrm{MHz}$ ) expected to be used for such data transmission. This, along with the tendency by the overhead wiring to pick-up interfering signals since there is no shielding tends to limit the reach of the medium voltage loop though repeaters can be used to extend this range. Although there are some rather gratuitous claims that power-line transmission may be able to extend the reach of broadband to rural areas, it is likely that this technology, if proven to work adequately, will compete with cable technologies in areas with rather high population density.

Some of the systems are to use the low voltage loop direct to the subscriber's house, allowing 
broadband connection through the AC outlets. This creates even more challenges with the electric noise generated by any brush electric motors such as those found in vacuum, hair dryer or fan on the same circuit. The local power network is also laid out in branches, with the same wires feeding many customers. That, as well as a collision course of capacitors, switches and other gadgets, will impact on the signal availability.

Reports indicate raw data speeds of up to 45 Mbit/s with real throughputs of approximately 18 Mbit/s to be shared among subscribers on the same medium-voltage circuit. Distance that can be covered on the medium-voltage circuit is about $1.6 \mathrm{~km}$.

All the effects enumerated above are well known and it is expected that, because of the potential market, the various companies will put a lot of effort in optimizing their technology to alleviate these drawbacks ${ }^{[7,8]}$. The impact will likely be translated in reduced effective throughput and variable availability. An area where the companies are not likely to put as much effort is on the interference aspect, especially interference to other services operating in the same region of the spectrum.

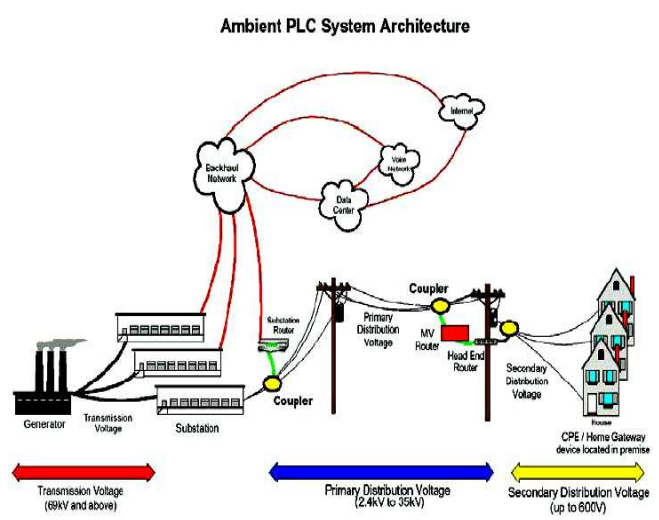

Fig 1: Power line Communication System Architecture 3.0 NOISE AND INTERFERENCE IN PLC

Noise is usually composed of randomly occurring voltages, which are unrelated in phase or frequency and sometimes consists of very peak in nature. Interference on the other hand is usually more structured than noise since it arise as unwanted coupling from just a few signals in the network.

\subsection{NOISE}

A power line communication network consists of a wired link and a wireless link. The wired link is consists of a pear to pear fiber optical network, with its normal noise and attenuation. The wireless portion is mainly affected by all the noises and alternation of free space/atmosphere. A power line network with associate noise is shown below.

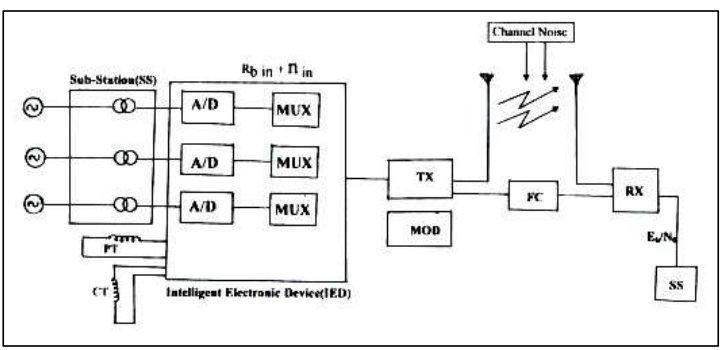

Fig 2: Power Line Communication Network

For the output (in the receiver, fig. 1) the bit error rate is given by $[2,3]$,

$$
B E R=f\left(\frac{E_{b}}{N_{o}}\right)
$$

Here $\mathrm{N}_{0}$, the output noise consists of channel fading, channel noise and Interferences, $E_{b}$ is the bit energy.

$$
\text { Again } \begin{aligned}
B E R & =0.5 \operatorname{erfc}\left[\sqrt{\frac{\gamma}{2}}\right] \\
\frac{E_{b}}{N_{o}}= & \frac{P_{S} T_{b}}{N_{o}} \\
& =\frac{P_{S}}{N_{o}\left(\frac{1}{T_{b}}\right)} \\
& =\frac{P_{S}}{N_{o} R_{b}} \\
& =\frac{P_{S}}{\sigma_{n}{ }^{2}}
\end{aligned}
$$

Here, $\quad \gamma=$ Signal to Noise ratio (SNR)

$\mathrm{P}_{\mathrm{s}}=$ Signal power

$\mathrm{T}_{\mathrm{b}}=$ Signal period

(Considering AWGN in the channel)

Now the total noise variance,

$\sigma_{n}^{2}=\sigma_{A W G N}^{2}+\sigma_{p s}^{2}$

Where, $\sigma_{p s}{ }^{2}=$ noise variance of the wired link.

and ${\sigma_{A W G N}}^{2}=$ noise variance of the wireless link

Now,

$$
\begin{aligned}
& \gamma_{o}=S N R \\
& =\frac{P_{S}}{\sigma_{n}^{2}+\sigma_{L}{ }^{2}}
\end{aligned}
$$

Where, $\quad \sigma_{n}^{2}=\sigma_{A W G N}^{2}+\sigma_{p s}^{2}=$ Total variance and $\sigma_{L}^{2}=$ Power line noise variance 


$$
\begin{aligned}
& \gamma_{0}=\frac{P_{S} / \sigma_{n}^{2}}{\left[1+\left(\frac{\sigma_{L}^{2}}{\sigma_{n}^{2}}\right)\right]} \\
= & \frac{\gamma}{\left[1+\frac{\sigma_{L}^{2}}{\sigma_{n}^{2}}\right]} \\
= & \frac{\gamma}{\left[1+K_{n}\right]}, \quad k_{n}=\frac{\sigma_{L}^{2}}{\sigma_{n}^{2}}
\end{aligned}
$$

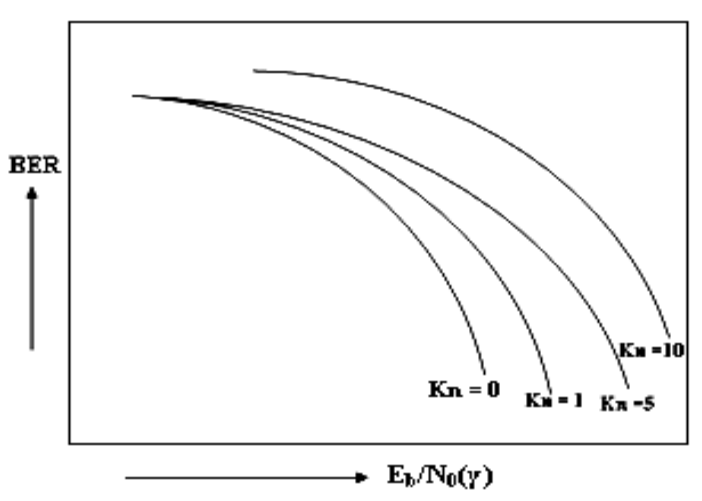

Fig 3: Variation of BER with $E_{b} / N_{0}$ for different values of $\mathbf{K}_{\mathbf{n}}$.

For a power line communication the noise is assume to be cyclostationary i.e. periodically stationary. So, its mean is zero and variance is synchronous to the AC voltage of the mains. The probability density function (PDF) of such noise at time $t$ is given by,

$$
P(n(t))=\frac{1}{\sqrt{2 \pi \sigma_{n}{ }^{2} t}} \exp \left[\frac{-n^{2} t}{2 \sigma_{n}{ }^{2} t}\right]
$$

This equation can be taken as a basic equation to model power line noise. In our subsequent paper we discussed thoroughly power line noise model in AWGN environment.

\subsection{INTERFERENCE}

It is likely that extensive signal processing will be used by industry to make the transmitted signal as robust as possible to impairments from the power grid and local power distribution in the houses ${ }^{[4]}$. This will make the signal robust ingress interference such as that coming from $\mathrm{TV}$, radio and mobile (police radios) communication system transmissions and even garage door openers. It is not clear, however, whether the industry will invest as much energy toward studying interference created by PLC that could affect other communication services operating in the same frequency range $(2-80 \mathrm{MHz})$. In the High Frequency (HF) band (3-30 MHz), a number of communication systems could be affected: HF broadcasting, radio-communications (fixed and mobile) services and amateur radio. Other services in the low VHF band will also need to be protected such as TV broadcasting and fixed and mobile services. The RF signals generated by PLC will easily radiate from the medium voltage overhead wiring and the local AC wiring (except in the case of the Amperion system that utilizes Wi-Fi to bring the signal to homes. The extent of these unintentional radiated emissions will depend on many factors, including differences in the configuration of the AC mains supply systems and the physical layout of the wiring. The management of unintentionally radiated emissions from PLC systems will need to be addressed under the inter-service interference limits and the electromagnetic compatibility (EMC) rules.

\subsection{Power line Communication Over High Voltage Lines}

Recently a study in Japan has been made to realize automatic reconfiguration of power distribution networks, data and commands are superposed on 6600 volts power lines. The structure of that 6600 volts power line is shown in fig 4 . below $[10,12]$.

Under normal circumstances the section switches (a e) are kept 'ON' and the interconnection switch (h) is kept 'OFF'. When the power line is short circuited in point $A$, the replay cuts off the power line and at the same time switches a, b, c, d and e are turned OFF. In a short period, relay $\alpha$ and switches $\mathrm{a}, \mathrm{b}$ and $\mathrm{c}$ turn $\mathrm{ON}$ sequentially When switch c turns on relay $\alpha$ detects the over current again and the short circuit between $\mathrm{c}$ and $\mathrm{d}$ can be located. At that time relay $\alpha$ and switches $a, b$ and $c$ turn off again. Then relay $\alpha$ and switches $\mathrm{a}, \mathrm{b}$, e and $\mathrm{h}$ are turn ON. Therefore only those consumers connected to the power line between switches $\mathrm{c}$ and $\mathrm{d}$ are not supplied with power, but all the other consumers are supplied with.

This process of reconfiguring the distribution network operates automatically so as to shorten the power failure time and minimize the area affected. In this case, PLC technology is used to transmit the data concerning the switches and the commands for controlling switches. 


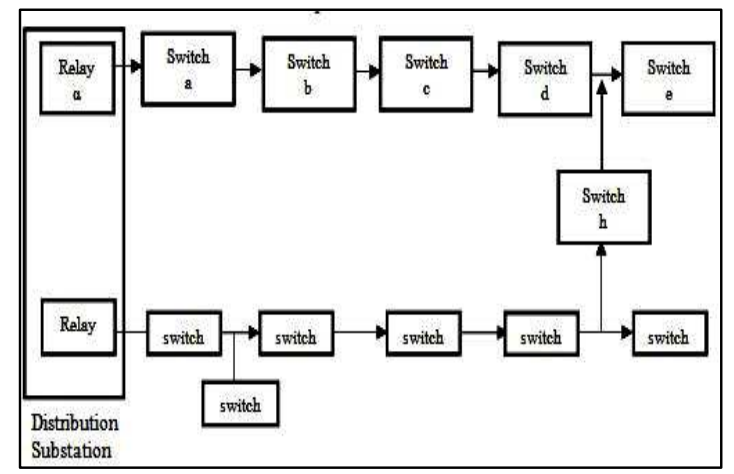

Fig 4: Connections between high voltage distribution lines

The data transmission has the following features:

- Data: Electricity current, status of which (ON or OFF); switch control command

- Transmission control: half duplex, polling system

- Frequency shift: $\pm 100 \mathrm{~Hz}$

- Output power : 10 watts

- Modulation : Frequency Shift keying (FSK)

- Data speed : 200bit/s

- Carrier:5040Hz+7440Hz;5280Hz+7680hz;...

$\ldots 7200 \mathrm{~Hz}+9600 \mathrm{~Hz}$

- Transmission length: up to $10 \mathrm{~km}$

- Power line coupling : shown in figure 5

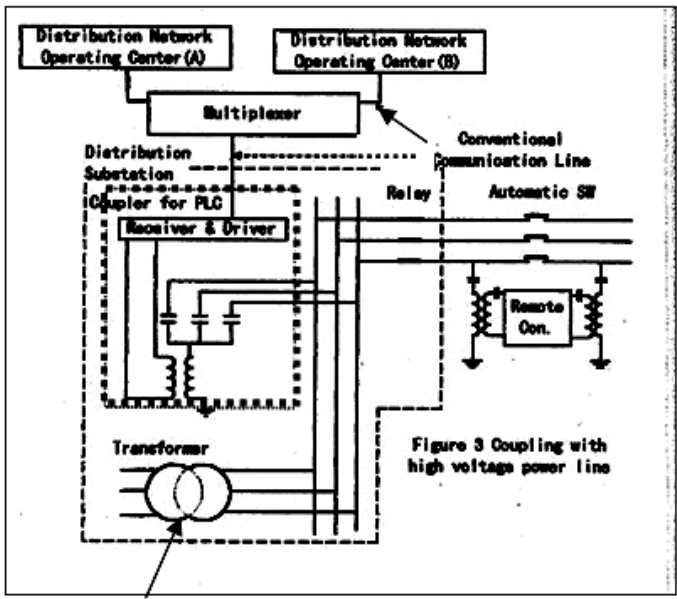

Fig 5: Coupling with high voltage power line

A study is also going over the power line communication through low voltage lines. The authors propose the same study for Bangladesh. With the assistance of appropriate authority and support this can be accomplished in MIST laboratory.

\subsection{NEED FOR PLC IN BANGLADESH}

Many ideas have been proposed concerning power-line applications as shown below. The authors strongly believe the following the same Bangladesh can move forward to a position of digital Bangladesh. Several novel proposals ${ }^{[12]}$ are:

\subsection{INDOOR USE}

i. For home application the proposed uses are:

- First aid information system use

- Remote control system switched by telephone

- Remote supervise of Mail \& Newspaper delivery post

- Electric key

- Interphone system

- Interphone system connected to telephone ')

- Time data transmission to electric appliances

- Home electric appliances switch control system

- Utilization of power-line radiation radio wave

- Home bus TV controller

- VTR timer remote reservation system

- VTR remote control

- Interlocking of TV \& VTR electric switches

- Signal transmission from amplifier to speaker

- Air conditioner remote control

- Ventilator remote control

- Boiling pot re-boiling switch remote control system

- Bath hot water supply switch remote control system

- Gas \& Water switch indicator 5'

- Window, window curtain remote control system

ii. Office use.

- Positioning indicator system

- Personal computer to printer transmission

- Power line facsimile transmission

- Indoor telephone

- Remote lock/unlock automated door system

- Other data transmissions 


\section{iii. Hotel use.}

- Refrigerator drink check system

- Emergency refuge guiding system

- Security check

- Restaurant waiter/waitress calling system

\section{iv. Factory use.}

- Motor remote control system

- Supervise of machine working time

- Welding machine control

- Protection of machine fault caused by voltage drop

\section{v. Misc use.}

- Clock remote control

- Light remote control system

- Disaster prevention system(fire sensor, smoke sensors)

- Rain shower warning systems

- Vending machine remote supervising systems

\subsection{OUTDOOR USE}

- Railway apparatus and ticket checking controlling

- Crane control system

\subsection{INTER F ACILITY SYSTEMS}

- Electric notice board system

- Public address systems

- M.C.A system for inside premises uses

- Temperature and humidity supervise system in a container ship

- Temperature and humidity supervise system in a vinyl pent houses

\subsection{Power Distribution Line Controlling}

- Electric switch supervising systems

- Miscellaneous remote controlling systems

\subsection{EXPLANATION OF THE USAGES}

Short explanations are given to those seems to unfamiliar to the reader in the following ${ }^{[13]}$ :

1. An information is transmitted to the indoor unit when something is delivered into the mail box in the outdoor.
2. Telephone and interphone functions are served by one terminal unit. An extension unit can be used to answer the calling guest.

3. Electric appliances transmit signals indicating their operational state through the power-line. A portable receiver detect roughly classified as follows. The signals radiated from the power-line and indicate the received information.

4. When watching VTR, TV channel switch turn on automatically and change channel to VTR automatically.

5. Electrically connect the Gas \& Water meters to an indicator arranged in the entrance room.

6. A cock operates a remote switch to transmit a dish ready signal to an indicator and the waiter/waitress observes it.

7. Several computers are connected to one keyboard and mouse in common and remotely switched. Computer monitors are settled several meters apart from the keyboard and the mouse.

8. A display board in which display figures can be electrically renewed.

9. Data generated each room meter are transmitted to a memory and from the memory a multiplexed signal is transmitted to the AMR center

\subsection{Conclusions}

As our main concern for electrical utilities is to decrease the power consumption peak and shift it to the low load hours. A communication system linking consumers and electric utilities is likely to prove particularly helpful in areas such as metering and the lowering of peak loading. The PLC net work as seen in the simplifies and reduces living expenditure as technology advances. Such kind of systems can be realized by some combination of fiber optics, CATV, the public subscriber telephone system and last but no the least PLC. We must continue to search for a better solution hence the BEST SOLUTION one that fits with the variety of business scenarios. While studying technical possibilities we will have to build up those business scenarios as they are for commercial purposes. We look forward for patronizing from the appropriate authority for our new vision "digital Bangladesh". 


\section{REFERENCES}

[1] M. Zimmermann and K. Dostert, "Analysis and modeling of impulsive noise in broad-band powerline communications," IEEE Trans. Electromagn. Compat., vol. EMC-44, no. 1, pp. 249-258, Feb. 2002.

[2] A Mathematical Model of Noise in Narrowband Power Line Communication Systems: Masaaki Katayama, Senior Member, IEEE, Takaya Yamazato, Member, IEEE, and Hiraku Okada, Member, IEEE: IEEE JOURNAL ON SELECTED AREAS IN COMMUNICATIONS, VOL. 24, NO. 7, JULY 2006.

[3] M. Katayama, S. Itou, T. Yamazato, and A. Ogawa, "Modeling of cyclostationary and frequency dependent power-line channels for communications," in Proc. 4th Int. Symp. Power Line Commun. Appl., Apr. 2005, pp. 123-130.

[4] A. Voglgsang, T. Langguth, G. Koerner, H. Steckenbiller, and R.Krnorr, "Measurement, characterization and simulation of noise on powerline channels," in Proc. 4th Int. Symp. Power Line Commun.Appl., Apr. 2000, pp. 139-146.

[5] H. Meng, Y. L. Guan, and S. Chen, "Modeling and analysis of noise effects on broadband power line communications," IEEE Trans.PowerDel., vol. 20, no. 2, pp. 630-637, Apr. 2005.

[6] Massaki KATAYAMA, "Introduction to Robust, Reliable and High-Speed Power-Line Communication Systems" IEICE trans. Fundamentals, VOL E84-A, no-12, Dec 2001.

[7] M. Tanaka and R. Someya, "The noise characteristics and transmission characteristics on data transmission using power line," IEICE trans., VOL J69-B, no-10, pp.1147-1149, Oct. 1986.

[8] S. Tachikawa, H. Hokari and G. Marubayashi,"Power line data transmission," IEICE Technical report, SSTA89-7, March 1989.

[9] O.C Hooijen, "A channel model for the lowvoltage power line channel: measurement and simulation results" Proc. 1997 Internatonal Symposium on Power line Communications and its Applications, pp.51-56,1997.

[10] Ezio Biglieri, Stefeno Galli, Yong-Hwan Lee, H. Vincent Poor, A. J. Han Vinck and W. H. Tranter,"Power Line Communication" guest editorial, IEEE jounnal on selcted areas in Communications, Vol.24, no.7, July 2006.

[11] Mr. Kazuma Takeshita et. Al, Fundamental study on application of spread spectrum communication systems to power distribution line carrier control (Part
3 \& Part 4);Central research institute of Electric power industry report(R91031,R92003.

[12] Juuji Ibuki, Power Line Communication In Japan Tokyo Electric Power Company 4-1 Egasm-Cno,T Ssurlomi-Kuy;O Kohillma 230-8510,Japan

[13] Yasuhito KAIZAWA, Gen MARUBAYASHI "Needs for the Power Line Communications" Institute for Information Systems Science, Soka University 1236 Tango-cho, Hachioji, Tokyo 192-0003, JAPAN 